\section{P15 ANTI-CARBAMYLATED PROTEIN ANTIBODIES' LEVELS ARE NEGATIVELY CORRELATED WITH CIRCULATING EFFECTOR T-CELLS IN A COHORT OF PATIENTS WITH SYSTEMIC LUPUS ERYTHEMATOSUS}

1,2Silvia Piantoni, ${ }^{1}$ Illaria Cavazzana, ${ }^{1}$ Francesco Poiatti, ${ }^{1}$ Stefania Masneri, 1,2 Roberta Ottaviani, ${ }^{1,2}$ Micaela Fredi, ${ }^{1,2}$ Franco Franceschini. ${ }^{1}$ Rheumatology and Clinical Immunology Unit, ASST Spedali Civili Brescia, Brescia; ${ }^{2}$ Dept. of Clinical and Experimental Sciences, University of Brescia, Brescia, Italy

\subsection{6/lupus-2020-eurolupus.64}

Background/Purpose Anti-carbamylated protein antibodies (antiCarP) were detected in a large cohort of patients with Systemic Lupus Erythematosus (SLE) in correlation with erosive arthritis but not with disease activity indexes. In animal models, T-cells may be activated by carbamylated epitopes playing a role in the development of arthritis. The imbalance between circulating regulatory (Treg) and CD28- effector T-cells was described in active SLE patients, explaining its involvement in disease's pathogenesis. Actually, no data are available about the possible correlation with these T-cell subpopulations and anti-CarP levels in SLE.

Methods Eight SLE patients with a median (10th-90th percentile) SLEDAI- $2 \mathrm{~K}=0 \quad(0-4)$, anti-dsDNA levels=34.1 (15.6427.4) $\mathrm{UI} / \mathrm{ml}(\mathrm{nv}<7), \mathrm{SDI}=1 \quad(0-1.3)$ were enrolled. Serum anti-CarP levels were evaluated using a home-made ELISA $(\mathrm{nv}<340 \mathrm{AU} / \mathrm{ml})$ and peripheral blood $\mathrm{T}$ cell immunophenotyping was done using Flow Cytometry (Beckman Coulter). Treg were defined as CD4+CD127lowCD25high T-cells.

Results Enrolled patients showed levels of anti-CarP $=189.38$ (93.5-341.1) AU/ml, Treg=2.2 (\% of CD4+), CD4+CD28$=7.7(4.8-22.5)(\%$ of CD4+) and CD8+CD28-=30.3 (17.235.6) (\% of CD8+). Analyzing possible correlations among different T-cell subtypes and anti-CarP levels, a significant inverse correlation was found between these autoantibodies and CD4 $+\mathrm{CD} 28$ - $\mathrm{T}$ cells $(\mathrm{r}=-0.8, \mathrm{p}<0.01$; Spearman rank correlation). No correlations were found between autoantibodies and other T-cell subpopulations or disease activity/damage indexes.

Conclusions In a small cohort of patients with serologically active SLE, anti-CarP autoantibodies were found as negatively correlated to circulating CD4+CD28- T-cells, which were described in association with disease damage, independently of age, gender, disease duration and activity. This suggest a potential role of anti-CarP as marker of SLE with a minor extent of T-cell activation and, consequently, with a possible better prognosis.

\section{P16 THE COMBINED TYPE-I INTERFERON AND NEUTROPHIL GENE SCORES IDENTIFY HIGHLY ACTIVE SYSTEMIC LUPUS ERYTHEMATOSUS PATIENTS AND PERFORMED BETTER THAN CLASSICAL SEROLOGICAL MARKERS}

${ }^{1}$ François Chasset, ${ }^{2}$ Camillo Ribi, ${ }^{3}$ Marten Trendelenburg, ${ }^{4}$ Uyen Huynh-Do, ${ }^{1}$ Pascale RouxLombard, ${ }^{5}$ Delphine S Courvoisier, ${ }^{1}$ Carlo Chizzolini. ${ }^{1}$ Dept. of Pathology and Immunology, School of Medicine University of Geneva, Geneva; ${ }^{2}$ Division of Immunology and Allergy, University Hospital Center of Lausanne, Lausanne; ${ }^{3}$ Dept. of Biomedicine and Division of Internal Medicine, University Hospital of Basel, Basel; ${ }^{4}$ Division of Nephrology and Hypertension, Inselspital, Bern University Hospital, Bern; ${ }^{5}$ Division of Rheumatology, University Hospital and School of Medicine, Geneva, Switzerland

10.1136/lupus-2020-eurolupus.65

Background In SLE, heterogeneous clinical expression and activity may reflect diverse pathogenic and/or effector mechanisms. We dwelled into SLE heterogeneity by assessing the expression of three gene sets representative of type I interferon (IFN-I), neutrophil-(PMN) and plasmablast-(PB) signatures in a well characterized, multidisciplinary cohort of SLE patients. We further assessed whether individual gene products could be representative of these three signatures.

Methods Whole blood, serum and clinical data were obtained from 140 SLE individuals. Gene expression was assessed by NanoString $@$ technology, using a panel of 37 probes allowing the computation of six IFN-I, one PMN and one PB scores. Protein levels were measured by ELISA.

Results High IFN-I gene expression was found in 45 to $50 \%$ of SLE individuals, depending on the score used. All 6 IFN-I scores were significantly associated with active skin involvement and 2 of 6 with arthritis. Interferon-induced GTP-binding protein $\mathrm{Mx} 1$ (MX1) correlated with IFN-I score $(\mathrm{p}<0.0001)$ and was associated with a similar clinical phenotype. High PMN gene expression was found in $25 \%$ of individuals in association with SLE fever, serositis, leukopenia and glucocorticoid use. PB gene expression was highly influenced by immunosuppressant agents with no association with SLE features. The combined IFN-I and PMN gene expression was significantly associated with high disease activity and outperformed anti-dsDNA, anti $\mathrm{C} 1 \mathrm{q}$ and complement levels to predict SLE activity.

Conclusions The IFN-I and PMN gene scores segregate with distinct SLE clinical features and their joint expression identify high disease activity. MX1 protein levels perform similarly to IFN-I gene expression.

Acknowledgements Work partially supported by funds provided by a grant from Fondation Fleurette Wagemakers, Sion (Switzerland), by a grant from La Société Académique de Genève (Switzerland). FC was supported by a research travel grant from the French Society of Dermatology and from Institut Servier, Paris (France).

\section{P17 ANTI-NEURONAL ANTIBODIES IN PATIENTS WITH SYSTEMIC LUPUS ERYTHEMATOSUS: PREVALENCE, CLINICAL ASSOCIATIONS AND SEARCHING FOR A SPECIFIC TARGET}

${ }^{1}$ Elisabetta Chessa, ${ }^{1}$ Maria Maddalena Angioni, ${ }^{1}$ Silvia Pinna, ${ }^{1}$ Mattia Congia, ${ }^{1}$ Alberto Floris, ${ }^{2}$ Elias Manca, ${ }^{2}$ Giulia Corda, ${ }^{3}$ Lorena Lorefice, ${ }^{2}$ Gian Luca Ferri, ${ }^{2}$ Cristina Cocco, ${ }^{1}$ Alessandro Mathieu, ${ }^{1}$ Alberto Cauli, ${ }^{1}$ Matteo Piga. ${ }^{1}$ Reumatologia, Policlinico Universitario AOU e Università di Cagliari, Cagliari; ${ }^{2}$ NEF-Lab, Dipartimento di Scienze Biomediche, Università di Cagliari, Cagliari; ${ }^{3}$ Neurologia, Ospedale Binaghi, ATS, Italy

\subsection{6/lupus-2020-eurolupus.66}

Background The aim of this study was to evaluate the prevalence and clinical significance of Anti-neuronal antibodies $(\mathrm{AnAb})$ in a cohort of neuropsychiatric (NP) Systemic Lupus Erythematosus (SLE) patients.

Methods Overall, 28 SLE patients (age $45.6 \pm 14.4$ years) with inflammatory neuropsychiatric syndromes, classified according to existing criteria $(1,2)$ were enrolled and serum samples were collected before starting treatment for the specific NP event; 41 consecutive SLE patients (44.2 \pm 14.2 years) without NP involvement, 22 Multiple Sclerosis patients (37.9 \pm 11.0 years) and 82 healthy subjects $(44.8 \pm 15.0$ years) served as controls. Search for AnAb was performed by tissue-based immunohistochemistry assays (IHC) on male Sprague Dawley rat's brain sections and confirmed by cell- 